\title{
Mandatory reporting legislation in Canada: improving systems for patient safety?
}

\author{
C. Milligan ${ }^{1,2 \star}$ (D), S. Allin ${ }^{1,2}$, M. Farr ${ }^{1,2}$, E. Farmanova ${ }^{2}$, A. Peckham ${ }^{1,2}$, J. Byrd ${ }^{3}$, R. Misfeldt ${ }^{3}$, \\ G. R. Baker ${ }^{1}$ and G. P. Marchildon ${ }^{1,2}$ \\ ${ }^{1}$ Institute of Health Policy, Management and Evaluation, University of Toronto, Toronto, Ontario, Canada, ${ }^{2}$ North American \\ Observatory on Health Systems and Policies, University of Toronto, Toronto, Ontario, Canada and ${ }^{3}$ Canadian Patient Safety \\ Institute, Ottawa, Ontario, Canada \\ *Corresponding author. Email: crystal.milligan@mail.utoronto.ca
}

(Received 3 March 2020; revised 6 January 2021; accepted 11 January 2021; first published online 18 February 2021)

\begin{abstract}
Patient safety is a complex systems issue. In this study, we used a scoping review of peer-reviewed literature and a case study of provincial and territorial legislation in Canada to explore the influence of mandatory reporting legislation on patient safety outcomes in hospital settings. We drew from a conceptual model that examines the components of mandatory reporting legislation that must be in place as a part of a systems governance approach to patient safety and used this model to frame our results. Our results suggest that mandatory reporting legislation across Canada is generally designed to gather information about rather than respond to and prevent - patient safety incidents. Overall, we found limited evidence of impact of mandatory reporting legislation on patient safety outcomes. Although legislation is one lever among many to improve patient safety outcomes, there are nonetheless several considerations for patient safety legislation to assist in broader system improvement efforts in Canada and elsewhere. Legislative frameworks may be enhanced by strengthening learning systems, accountability mechanisms and patient safety culture.
\end{abstract}

Key words: learning systems; legislation; mandatory reporting; patient safety

\section{Introduction}

Ensuring the safety of patients is an essential component of any effort to improve health system quality and performance (Donaldson et al., 2000). Estimated to be the 14th leading cause of global morbidity and mortality (Jha et al., 2013), patient harm represents a clinical and economic burden around the world (Slawomirski et al., 2017; Flott et al., 2019). Defined as the absence or reduction of risk of health care-associated harm (World Health Organization, 2019), patient safety was declared a global health priority by the World Health Assembly in May 2019 (Seventy-Second World Health Assembly, 2019). Although its current international importance is clear (National Academies of Sciences, 2018; Flott et al., 2019), patient safety remains challenging for health care systems to put into practice.

In Canada, despite increasing attention to health care quality and patient safety, the safety of patients in Canadian health care institutions has been and remains a public health concern (Baker et al., 2004; Pellegrini, 2014; RiskAnalytica, 2017). Patients continue to experience harm in approximately one out of every 18 hospitalisations (Canadian Institute for Health Information and Canadian Patient Safety Institute, 2016). Other estimates suggest that by 2047, infections alone will drive nearly $40 \%$ of all patient safety incidents in acute care settings, increasing health care costs by an average of $\$ 480$ million per year (RiskAnalytica, 2017).

(C) The Author(s), 2021. Published by Cambridge University Press. This is an Open Access article, distributed under the terms of the Creative Commons Attribution licence (http://creativecommons.org/licenses/by/4.0/), which permits unrestricted re-use, distribution, and reproduction in any medium, provided the original work is properly cited. 
The reporting of patient safety incidents is considered a critical component of international system efforts to improve patient safety (World Health Organization, 2009; Berwick, 2013; Frankel et al., 2017; OECD, 2019), yet national-level reporting in Canada is narrowly focused on medication or vaccination-related incidents and largely dependent on voluntary reporting by health care organisations (Boucaud and Dorschner, 2016). In the past decade, Canadian legislation has increasingly been used as a tool to strengthen patient safety (Erdmann, 2018). Notably, the Protecting Canadians from Unsafe Drugs Act, also known as Vanessa's Law, made incident reporting with regard to drug reactions and medical devices mandatory for all Canadian hospitals as of December 2019 (Health Canada, 2019). Although this is an important move by the federal government to strengthen the regulation of therapeutic products for patient safety, for constitutional reasons the majority of patient safety legislation in Canada, a decentralised federation, sits with the provincial and territorial levels of government. Variation among patient safety legislation across the provinces and territories provides a unique opportunity for comparative analysis and learning about the influence of legislated mandatory incident reporting on patient safety itself.

Considerable attention has been paid in the literature to the role of organisational policies and quality improvement initiatives to improve patient safety (Leape and Berwick, 2005; Wachter, 2010; Chassin and Loeb, 2013). However, the role of legislation, an important component of systems governance, has received less scrutiny. This paper presents the results of a study that addressed this gap. First, we describe what is known in peer-reviewed literature about legislation related to mandatory reporting of patient safety incidents and its influence on patient safety in hospital settings. Second, we explore the approaches taken by Canadian provincial and territorial governments to use legislation to mandate incident reporting as part of their patient safety improvement efforts. Our findings highlight a number of considerations to inform system improvements for patient safety in Canada and elsewhere.

\section{Legislation as a component of systems governance}

In systems thinking, problems must be understood in the context of interrelated, complex and dynamic systems (De Savigny and Adam, 2009). Thus, a systems governance approach views patient safety as an outcome of the entire health care system, which requires action at every level. Reason (1997) illustrated system error through the 'Swiss cheese model' wherein different layers of protection against preventable harm exist at multiple levels but, like Swiss cheese, each layer has holes. When the holes at different levels align, patients can be harmed.

Patient safety legislation can serve multiple roles in a systems governance approach to improve patient safety. Downie and colleagues (2006) proposed three objectives against which patient safety legislation can be assessed. First, the legislation should enable knowing about patient safety incidents through reporting, investigation, analysis and enquiry. Second, the legislation should allow us to respond to patient safety incidents through learning and accountability at both individual and systems levels. Third, the legislation should help to prevent incidents through the regulatory or policy framework that controls or influences care delivery.

On the basis of a comprehensive review and analysis of federal, provincial and territorial legislation in Canada, Baker et al. (2008) proposed 10 essential elements of legislation related to patient safety incident reporting that are necessary to support the development of learning systems (see Table 3). These essential elements identify specific components of legislation that should be in place as part of a systems governance approach to patient safety incident reporting.

Figure 1 illustrates how the concepts of knowing about, responding to, and preventing patient safety incidents can be combined with the 10 essential elements of legislation for patient safety incident reporting, creating a framework with which we can compare and assess the effectiveness of legislation in improving safety. This conceptual framework underscores a cycle of learning as the link between response and prevention of patient safety incidents. In other words, an effective response that includes thorough review of the patient safety incident and any lessons learned will 


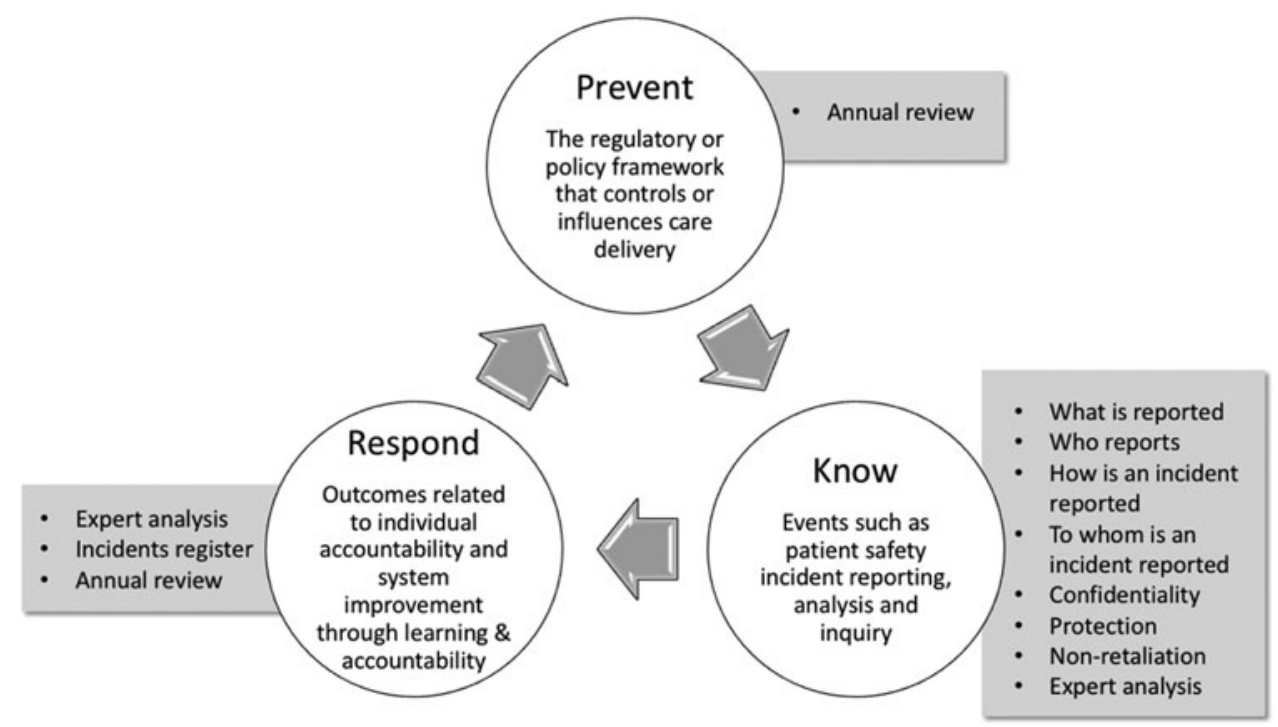

Figure 1. Patient safety system objectives (know, respond and prevent) and the essential elements of legislation for patient safety incident reporting. Adapted from Downie et al. (2006) and Baker et al. (2008). Details are provided in the Supplementary materials.

contribute to preventing similar incidents in the future. Establishing a knowledge base about patient safety incidents, which can be shared in support of individual and organisational learning, is critical to safer care delivery. It is through a cycle of learning - reporting and investigating incidents, identifying activities to mitigate impact and sharing lessons broadly - that additional similar incidents can be prevented (Baker et al., 2008; Incident Analysis Collaborating Parties, 2012).

\section{Methods}

Data collection took place between January and May 2019 and followed a multi-methods approach, including a scoping review and case study that included consultation with expert informants in senior health system leadership positions.

\subsection{Scoping review}

We conducted a scoping review to determine what is known in peer-reviewed academic literature about associations between mandatory reporting legislative and regulatory frameworks and patient safety outcomes in acute or hospital-based care settings. The scoping review followed methodology described by Arksey and O'Malley (2005). Insight gained through consultation with expert informants further deepened and contextualised the interpretation of preliminary findings (Levac et al., 2010) (Figure 2).

Between 18 and 20 February 2019, we searched three academic databases: Ovid MEDLINE, CINAHL Plus with full text and ProQuest Research Library. In consultation with an academic health sciences librarian, we searched Ovid MEDLINE first, using variations of key terms including 'patient safety', 'legislation' and 'mandatory reporting'. Our search yielded 231 results. We translated our strategy for searches in CINAHL Plus and ProQuest, which yielded 121 and 180 results, respectively. Our full search strategy is detailed in Table 1.

Articles had to meet specific eligibility criteria in order to be included in full-text review, namely: focus on an acute or hospital-based care setting; and examination of (or at least reference to) a law or regulation as well as some measure of a patient safety outcome. 


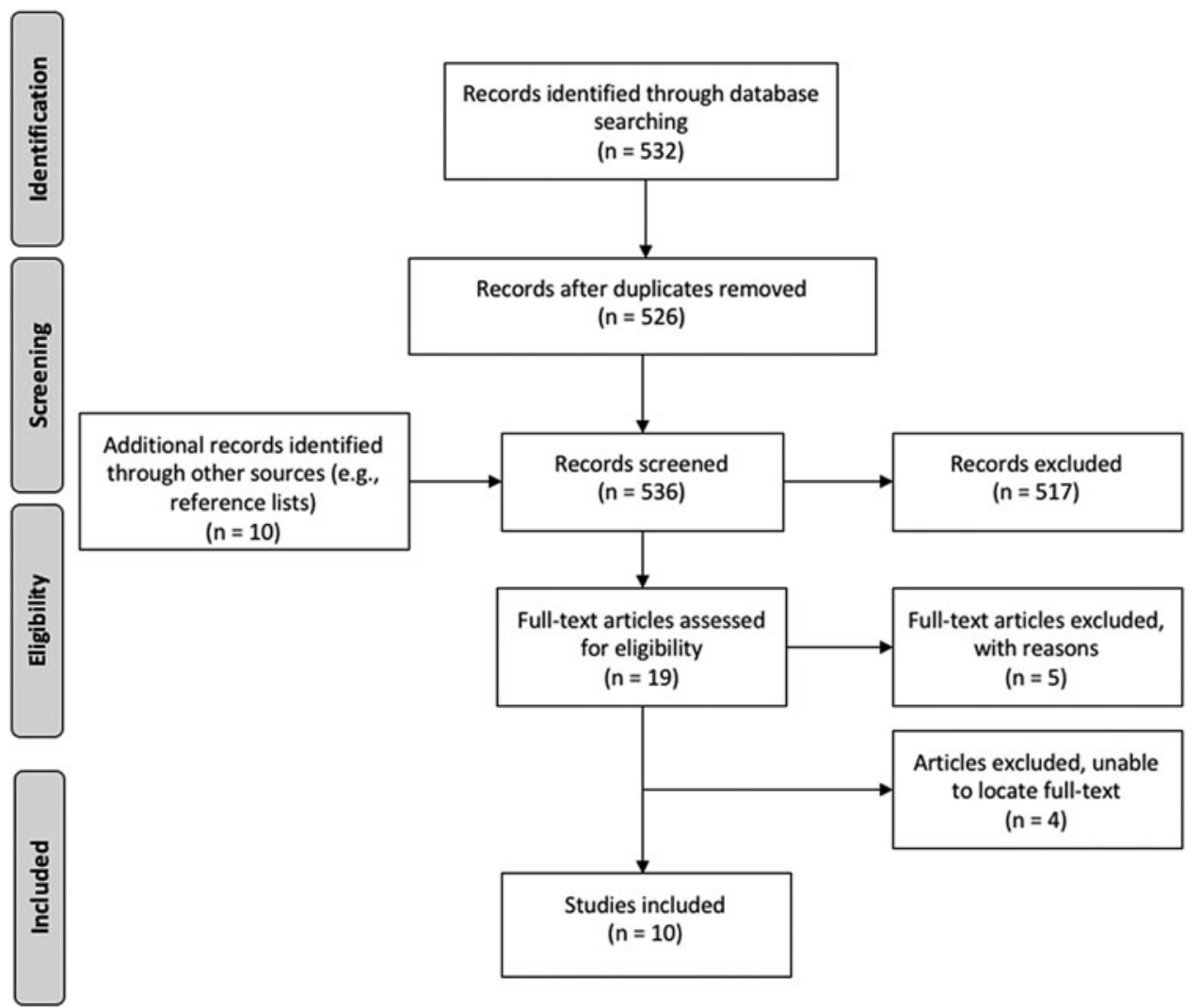

Figure 2. Scoping review study selection.

\subsection{Case study of Canadian mandatory reporting legislation}

In our case study, we compared provincial and territorial legislation pertaining to mandatory reporting of patient safety incidents. As a first step, we reviewed a report produced by the Canadian Patient Safety Institute (2018) that identified all legislation across Canada governing the reporting of patient safety incidents. Narrowing our scope to the eight Canadian jurisdictions that have instituted mandatory reporting legislation, we assessed each piece of legislation for provisions that relate to our conceptual framework. This assessment involved applying the concepts of knowing about, responding to, and preventing patient safety incidents (Downie et al., 2006) as well as the ten essential elements outlined by Baker et al. (2008) in order to assess the comprehensiveness of legislation, verifying the extent to which the legislation addressed each component of the conceptual framework. The final list of legislation that provided information within our framework and thus explicitly relates to mandatory reporting of patient safety incidents is found in Table 2.

We also consulted with expert informants working in safety and quality of care in jurisdictions across Canada. From 13 March to 31 May 2019, inclusive, consultation with 13 senior executives in three jurisdictions generated contextual and experiential evidence to clarify and understand the interpretation and implementation of legislation in practice, focusing particularly on the interpretation and use of provisions related to mandatory patient safety incident reporting. These consultations also helped to explore and clarify how legislation interacts with patient safety policies and initiatives at other system levels such as professional self-regulation. Although we prepared 
Table 1. Scoping review search strategy

\begin{tabular}{|c|c|c|}
\hline Database & Search strategy & Yield \\
\hline Ovid MEDLINE & $\begin{array}{l}\text { 'Patient Safety OR (outcome* OR indicator* OR data OR measure*)', 'Patient Safety', 'Patient Safety OR (Law OR regulation* OR Legal } \\
\text { OR Legislation OR Policy)', 'Legislation Hospital' and 'Mandatory Reporting OR Voluntary reporting' }\end{array}$ & 231 results \\
\hline CINAHL Plus & $\begin{array}{l}\text { 'Patient Safety+' OR 'Patient Safety/LJ' OR 'Patient Safety or (outcom or indicator* or Data or Measure*)' OR 'patient safety’, AND } \\
\text { 'Patient Safety OR Law* OR Regulation* OR Legal OR Legislation OR Policy', AND 'Legislation' OR 'Legislation, Hospital' OR } \\
\text { 'Legislation, Nursing' OR ‘Legislation, Medical' OR 'Legislation, Drug' OR 'legislation hospital', AND 'Mandatory Reporting' OR } \\
\text { 'Voluntary Reporting' }\end{array}$ & 121 results \\
\hline $\begin{array}{l}\text { ProQuest Research } \\
\text { Library }\end{array}$ & $\begin{array}{l}\text { 'Patient Safety Outcome' AND 'Patient Safety’ AND 'in-patient OR in-hospital OR patient OR hospital' AND 'Health care Legislation AND } \\
\text { Mandatory Reporting OR Voluntary Reporting' }\end{array}$ & 180 results \\
\hline
\end{tabular}


Table 2. Legislation reviewed for each jurisdiction with legislated mandatory reporting

\begin{tabular}{|c|c|}
\hline Jurisdiction & Provincial/territorial legislation \\
\hline British Columbia (BC) & $\begin{array}{l}\text { - Hospital Act Regulation, BC Reg } 121 / 97 \\
\text { - Evidence Act, R.S.B.C. 1996, c. } 124 \\
\text { - Designation Regulation, BC Regulation 363/95 }\end{array}$ \\
\hline Saskatchewan (SK) & $\begin{array}{l}\text { - The Provincial Health Authority Act, S.S. 2017, c.P-30.3, ss.8-2, 9-5(1)(aa) } \\
\text { - Critical Incident Regulations, 2016, R.R.S. c. R-8.2 Reg. } 10 \\
\text { - The Patient Choice Medical Imaging Act, S.S. } 2016 \text {, c. P-4.11, s. } 13\end{array}$ \\
\hline Manitoba (MB) & $\begin{array}{l}\text { - Regional Health Authorities Act, C.C.S.M. c. R34, Part } 4.1 \\
\text { - Critical Incidents Regulation, Man. Reg. } 211 / 2006 \text {, s.4 } \\
\text { - Manitoba Evidence Act, C.C.S.M. c. E150, ss. 9-10 }\end{array}$ \\
\hline Ontario (ON) & $\begin{array}{l}\text { - Excellent Care for All Act, 2010, S.O. 2010, c. 14, s. 8(2) } \\
\text { - Hospital Management Regulation (Public Hospitals Act), R.R.O. 1990, Reg. } \\
\text { 965, ss. 2(4)-(6), } 23 \\
\text { - Quality of Care Information Protection Act, 2016, S.O. 2016, c.6, Sch. } 2\end{array}$ \\
\hline Québec (QC) & - An Act respecting Health Services and Social Services, CQLR c. S-4.2 \\
\hline New Brunswick (NB) & - Health Quality and Patient Safety Act, S.N.B. 2016, c. 21 , s. 3 \\
\hline Newfoundland and Labrador (NL) & $\begin{array}{l}\text { - Patient Safety Act, S.N.L. } 2001 \text { c.P-3.01, s. } 17 \\
\text { - Evidence Act, R.S.N.L. } 1990 \text {, c. E-16, s. } 8.1 \\
\text { - Personal Health Information Act, S.N.L. } 2008 \text {, c. P-7.01 }\end{array}$ \\
\hline Northwest Territories (NT) & $\begin{array}{l}\text { - Hospital Insurance and Health and Social Services Administration Act, } \\
\text { R.S.N.W.T. 1988, c. T-3 } \\
\text { - Evidence Act, R.S.N.W.T. 1988, C. E-8, ss. 13-15 }\end{array}$ \\
\hline
\end{tabular}

questions and points for discussion in advance, these were open-ended conversations. The expert informants' contributions are integrated throughout Section 4 of this paper. Additional details related to our expert informants and the interviews are available in the Supplementary materials.

\section{Results}

\subsection{Scoping review}

Our academic database search yielded 526 citations after de-duplication. A citation review yielded 10 additional articles that were included in first-level title and abstract screening. In total, 19 peerreviewed articles met the criteria for full-text review. Of these, 10 articles met all eligibility criteria and therefore contribute to these results.

Most of the eligible studies were conducted in the United States (Stone et al., 2007, 2011, 2015; Linkin et al., 2013; Pakyz and Edmond, 2013; Marsteller et al., 2014; Woodward and Umberger, 2016), while one study was conducted in Canada (Daneman et al., 2012), one in France (Lucet et al., 2013) and another looked at England, France, Germany and the United States (Haustein et al., 2011). They examined the impact of legislation on health care-associated infections. Three focussed on central line-associated bloodstream infections (CLABSI) in hospital intensive 
care units (Pakyz and Edmond, 2013; Marsteller et al., 2014; Woodward and Umberger, 2016). Additional details on each article can be found in the Supplementary materials.

The impact of patient safety legislation (including mandatory and voluntary reporting laws) on patient safety outcomes varied across studies. Two articles reported a statistically significant association between mandatory reporting and a reduction in patient safety incident rates (Stone et al., 2011; Daneman et al., 2012). The remaining studies found limited impact (Woodward and Umberger, 2016); inconclusive evidence of impact (Stone et al., 2007, 2015; Haustein et al., 2011; Lucet et al., 2013) or no statistically significant impact (Linkin et al., 2013; Pakyz and Edmond, 2013; Marsteller et al., 2014). One article compared rates between mandatory reporting jurisdictions and voluntary reporting jurisdictions and found no difference in impact on outcomes (Stone et al., 2015).

Some studies described means by which legislation may incentivise organisational and health care provider behaviour to improve patient safety. These studies described collaboration and learning to reduce incident rates as particularly influential (Marsteller et al., 2014; Stone et al., 2015). Authors also argued that to improve patient safety outcomes, a combination of legislation and organisational change is needed, combined with an environment that promotes evidencebased processes and best practices for patient safety (Stone et al., 2011; Marsteller et al., 2014).

In five articles, the introduction of patient safety legislation was associated with a move towards an organisation-wide commitment to safety precautions and procedures (Haustein et al., 2011; Daneman et al., 2012; Lucet et al., 2013; Marsteller et al., 2014; Woodward and Umberger, 2016). For example, three articles drew attention to change in legislation as a possible motivator behind organisational and health care provider change to enhance patient safety through improved hand hygiene (Haustein et al., 2011; Lucet et al., 2013; Marsteller et al., 2014). Observing increased participation rates in one collaborative programme that aimed to reduce CLABSI rates and instil a patient safety culture, Marsteller et al. (2014) suggested that mandatory reporting legislation may incentivise peer learning, described as learning and sharing best practices between and among hospitals.

Marsteller et al. (2014) further stressed that because legislation will not teach health care organisations or systems how to reduce incident rates, there is a real need for peer learning as well as other deliberate actions to identify and remedy factors that contribute to patient harm, including resource allocation and the establishment of team tools or routines in support of information sharing. In other words, legislation may influence or create an incentive for hospitals to adopt best practices, but best practices are not often easy to implement. Thus peer-learning structures and collaborative programmes provide a means for hospitals to build, share and improve patient safety culture and best practices.

\subsection{Case study of Canadian mandatory reporting legislation}

In Canada, eight of 13 provincial and territorial jurisdictions have legislation pertaining to mandatory patient safety incident reporting. Table 3 summarises our assessment of each jurisdiction and depicts the variation in provincial and territorial legislative approaches to patient safety according to the 10 essential elements of legislation for patient safety incident reporting (Baker et al., 2008). The year that mandatory reporting legislation was introduced in each jurisdiction is indicated in the top row of the table.

\subsubsection{Knowing about patient safety incidents}

All eight jurisdictions have legislation that outlines the patient safety incident information that must be reported. Although the legislation includes clear definitions, these definitions do not necessarily align with international best practice. The World Health Organization (2009) defines a patient safety incident as an event or circumstance that resulted, or could have resulted, in unnecessary harm to a patient. In turn, there are three types of patient safety incidents: harmful 
Table 3. Assessment of legislation related to mandatory reporting of patient safety incidents: fulfilment (Yes or No) of 10 essential elements of legislation (Baker et al., 2008)

\begin{tabular}{|c|c|c|c|c|c|c|c|c|c|}
\hline \multicolumn{2}{|c|}{ Element } & \multirow{2}{*}{$\begin{array}{l}\text { BC (2013) } \\
\text { Yes }\end{array}$} & \multirow{2}{*}{$\begin{array}{l}\text { SK (2004) } \\
\text { Yes }\end{array}$} & \multirow{2}{*}{$\begin{array}{l}\text { MB (2005) } \\
\text { Yes }\end{array}$} & \multirow{2}{*}{$\begin{array}{l}\text { ON (2011) } \\
\text { Yes }\end{array}$} & \multirow{2}{*}{$\frac{\text { QC (2002) }}{\text { Yes }}$} & \multirow{2}{*}{$\begin{array}{l}\text { NB (2018) } \\
\text { Yes }\end{array}$} & \multirow{2}{*}{$\begin{array}{l}\text { NL (2017) } \\
\text { Yes }\end{array}$} & \multirow{2}{*}{$\begin{array}{l}\text { NT (2016) } \\
\text { Yes }\end{array}$} \\
\hline 1 & Detail on what is reported & & & & & & & & \\
\hline 2 & Detail on who makes a report & Yes & Yes & Yes & Yes & Yes & Yes & Yes & Yes \\
\hline 3 & Detail on how an incident is reported & Yes & Yes & Yes & Yes & Yes & Yes & No & No \\
\hline 4 & Detail on to whom an incident is reported & Yes & Yes & Yes & Yes & Yes & Yes & Yes & Yes \\
\hline 5 & Provisions for confidentiality & No & Yes & Yes & Yes & Yes & Yes & Yes & Yes \\
\hline 6 & Protections in legal proceedings & Yes & Yes & Yes & Yes & Yes & Yes & Yes & Yes \\
\hline 7 & Provisions for non-retaliation & No & No & Yes & Yes & No & Yes & Yes & No \\
\hline 8 & Provisions for expert analysis & No & Yes & Yes & Yes & Yes & Yes & Yes & Yes \\
\hline 9 & Mandated incidents register & No & No & No & No & Yes & No & No & No \\
\hline 10 & Mandated annual review & No & No & No & Yes & No & No & No & No \\
\hline
\end{tabular}

Notes: The assignment of Yes and No provides a high-level summary of the legislation in order to draw comparisons, but this approach masks some of the variation that is seen even among jurisdictions with the

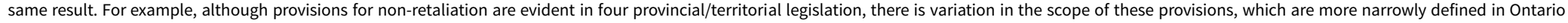
than in the other three jurisdictions. 
incidents (where incidents result in harm to a patient), no harm incidents (incidents that reach a patient with no discernible harm) and near misses (incidents that do not reach a patient and do not result in harm) (World Health Organization, 2009). Although New Brunswick (NB) was the only jurisdiction whose legislation was found to use the term 'patient safety incident' and provide a definition resembling that of the World Health Organization, legislation in the other jurisdictions used different terminology ('critical incident' was the most common term used) to express very much the same definition encompassing harmful incidents, no harm incidents and near misses. The exceptions to this were British Columbia (BC), Manitoba and Ontario, where definitions - 'critical incident' in Manitoba and Ontario, and 'serious adverse event' in BC - include neither no harm nor near miss incidents and instead focus solely on incidents where harm does occur.

Each jurisdiction has legislated who can make a report and to whom a report is submitted. Manitoba and Northwest Territories (NT) have the widest definition of who can report an incident. Although engagement with patients and their families is acknowledged as an integral component of incident analysis (Incident Analysis Collaborating Parties, 2012), these two jurisdictions are the only ones that include provisions enabling a patient or family member to report an incident under the same legislation as a person working for the health authority. Expert informants explained that patient safety incident reporting systems are usually limited to hospital settings, thus access to these reporting systems is limited to hospital-based health care providers. Despite the hospital-based focus of this study, our expert informants suggested that mandatory reporting legislation needs to be system-wide, not sector-based.

Except for Newfoundland and Labrador (NL) and NT, where legislation leaves room to develop more detailed regulations not yet in force, all jurisdictions have directed requirements for how an incident is to be reported. In many jurisdictions (BC, Manitoba, NB, NL and Québec) this direction is provided in minimal detail within legislation; rather, each individual health care organisation develops its own regulation or written procedures for reporting an incident.

There is also variation in the extent to which the laws that we reviewed protect people who provide information about patient safety incidents from personal liability, suspension, demotion, harassment and other retaliatory behaviour. Non-retaliation provisions in several provinces are wide-ranging and consistently worded. For instance, the Patient Safety Act of NL states that 'a person shall not dismiss, suspend, demote, harass or otherwise disadvantage or penalise a healthcare provider who reported a close call or an occurrence.' Among the jurisdictions with mandatory reporting legislation, such provisions are not in place in BC, Québec, Saskatchewan and NT.

Confidentiality and protection in legal proceedings are specified in nearly all the jurisdictions' laws, although protections are in place to varying degrees. For example, for information to receive protection in Ontario, it must be connected with a 'quality of care committee' designated under the Quality of Care Information Protection Act (QCIPA). As such, the protection appears to be very narrow. In contrast, the protection in Manitoba is quite broad and covers 'a notice, report or other record of information respecting a critical incident that is required to be provided by a health corporation, prescribed health care organisation or regional health authority'. BC is the only jurisdiction whose mandatory reporting legislation does not contain specific provisions for confidentiality. According to expert informants we spoke with from different jurisdictions, emphasis on confidentiality and privacy serves to constrain the sharing of information across organisations and authorities, resulting in an inability to learn system-wide about the factors that lead to patient harm.

Expert informants who represented front-line health care providers in BC and Ontario also identified their lack of real-time data as a constraint in their oversight of unsafe practices. They described access to system-level patient safety surveillance data, whether sourced from patient safety incident reports, administrative data or chart reviews, as particularly important to help health system stakeholders identify provincial and local safety champions and outliers 
and be able to intervene before incidents reoccur. They also argued that individual health care professionals require access to their own performance data so as to address any shortcomings in their practice and do their part to contribute to improved patient safety.

\subsubsection{Responding to and preventing patient safety incidents}

Ontario and Québec are the only jurisdictions whose legislation requires annual incident reviews or a register of patient safety incidents. Legislation in Ontario mandates aggregate patient safety incident reviews at least twice a year in every public hospital but does not mandate a province-wide incident register. Instead, Ontario hospitals maintain their own incident data in hospital-level registers from which the Ministry of Health may request information. In Québec, a provincial register is managed by the Ministry of Health, which may enable the monitoring and analysis of incidents at the system level, but there is no mandated annual review to facilitate learning for system improvement.

Although protection in legal proceedings is narrowly focused on information put forward by a 'quality of care committee', Ontario's framework appears to be the most comprehensive as it covers all but one of the essential elements suggested by Baker et al. (2008). Still, some expert informants in Ontario suggested that rigid mandatory reporting requirements encourage 'gaming' of the system to artificially reduce incident rates. In comparison, much of the responsibility for regulating and implementing patient safety in $\mathrm{BC}$ is delegated to professions and organisations within the regional health authorities. $\mathrm{BC}$ legislation covers fewer essential elements than does legislation in any of the other jurisdictions with mandatory reporting. Expert informants in BC described their approach as having empowered professional regulatory bodies to identify system factors that lead to harm, and to participate in collaborative policy making. These $\mathrm{BC}$ expert informants also felt that regardless of legislation, organisational-level initiatives and policies were important contributors to system improvements for patient safety.

One BC initiative introduced in 2013 is a robust patient safety learning system (PSLS) that remains the only province-wide system of its kind in Canada. Accessible within health authority firewalls, the PSLS uses real-time digital information and technology to facilitate reporting and learning from patient safety incidents across regional health authorities. It is co-owned by all regional health authorities in the province and run by a team governed by a provincial steering committee with senior-level representation from each authority. The PSLS is therefore able to work closely with authorities and organisations in a way that supports investigation of a reported incident, sharing of information and learning across the provincial system, and promotion of a culture of patient safety. Acknowledging an ongoing need for the PSLS steering committee and staff to support health authorities to recognise patient safety incidents and see value in reporting them, expert informants in $\mathrm{BC}$ nonetheless attributed incident reporting to organisational culture and policy, not mandatory reporting legislation. They described the PSLS as a system that exceeds legislated reporting requirements in its ability to support learning.

Overall, there was agreement among expert informants involved in this study that legislation plays an important role in creating an environment conducive to patient safety, although it has limited direct impact.

\subsubsection{Emergent themes: accountability, communication and culture}

Three noteworthy themes emerged from our consultation with expert informants: accountability, communication and culture. First, the expert informants expressed concern about health care providers not being held accountable for their adherence to professionally accepted standards of safety. The Public Hospitals Act in Ontario was cited by one expert informant as a specific piece of legislation that could be amended to reduce legal deterrents to hospital oversight of physician performance. Some expert informants suggested a need to strengthen the alignment between professional regulation, policy and patient safety legislation by way of increasing the accountability of professional colleges to government. For instance, it was noted that routine 
reporting of patient safety data to government by provider organisations and professional regulatory colleges could strengthen the accountability of providers for patient safety.

Second, according to our expert informants, the ability of health system actors such as hospitals, regulatory bodies and quality councils to identify and respond to, learn from and prevent patient safety incidents is hampered by silos and unclear channels of communication. Numerous expert informants suggested that leadership on this issue should come from the federal or pan-Canadian level, with multilevel and multisectoral collaboration to develop meaningful definitions and indicators for patient safety, as well as infrastructure to support data collection and access. Cross-jurisdictional communication and collaboration were seen to be complicated by the various definitions and conceptualisations of patient safety that exist across the country.

Third, the expert informants suggested that although legislation is clearly designed to encourage reporting and analysis of incidents, the lack of a well-developed organisational patient safety culture limits what is known about patient safety incidents. In the words of one expert informant, patient safety incident reporting in the absence of a strong patient safety culture is 'like you're ratting people out'. Our expert informants suggested a need to foster a culture of sharing and learning for patient safety before legislating mandatory reporting. Support for this culture across organisations, systems and professions, they said, is a decisive facilitator in successfully responding to and preventing patient safety incidents.

\section{Discussion}

In light of a shortfall in patient safety studies that investigate the associations between legislation and outcomes, there is limited evidence of direct, quantifiable impact of mandatory reporting legislation on patient safety outcomes. Nonetheless, our case study of provincial and territorial legislation in Canada sheds light on the characteristics of a legislative framework that would support patient safety.

In the majority of Canadian jurisdictions, mandatory reporting legislation falls short of providing mechanisms for learning and preventing patient safety incidents. Effective learning systems are characterised by continuous prospective and retrospective self-reflection and identification of strengths and weaknesses, including action to address weaknesses or gaps (Frankel et al., 2017). They combine local reporting and analysis with data sharing at national and sub-national levels (Baker et al., 2008). By contrast, legislation related to privacy and confidentiality presents a challenge to enabling the sharing of data and learning across institutions and jurisdictional borders. Confidentiality obligations can, in fact, serve to limit sharing of useful incident information such as the characteristics of a patient that must be reported and understood to prevent future incidents (Baker et al., 2008).

Furthermore, there have been limited efforts by Canadian governments to create and build PSLSs. For example, in Ontario, the provincial government accepted a 2014 recommendation to develop a mechanism for hospitals to share what they learn from their investigations of patient safety incidents (Morin and Laupacis, 2014), yet there has been no discernible movement towards developing this system. Indeed, some observers view the Ontario regulatory oversight structure as outdated and incapable of effectively monitoring professional practice (Waddell et al., 2017). This situation exists across jurisdictions, including those with mandatory reporting legislation, despite a perception that mandatory reporting serves as a system of accountability (Health Quality Council of Alberta, 2017).

Matters are complicated by a general paucity of standardised patient safety data within and across provinces and territories. The Canadian Institute for Health Information's National System for Incident Reporting is based on voluntary reporting and thus cannot be used to measure rates of patient harms within or across jurisdictions (Canadian Institute for Health Information, 2019). Although the recent collaborative project between the Canadian Patient Safety Institute and the Canadian Institute for Health Information to measure hospital harm marks a major 
step forward in the standardised measurement of hospital-based patient safety in Canada (Canadian Institute for Health Information and Canadian Patient Safety Institute, 2016), the data are not publicly available at the hospital, provincial or territorial levels and thus are not widely used in patient safety literature or research efforts (these data are available only to provincial and territorial governments, health authorities and hospitals). Regular public reporting of standardised data has the potential to support peer learning, system-wide monitoring and planning and comparative research. On the international stage, the Global Patient Safety Alerts initiative, a growing online collection of publicly available, indexed patient safety incidents worldwide, aims to promote cross-jurisdictional learning and transparency (Canadian Patient Safety Institute, 2016a).

Certainly, a common language for patient safety would allow for greater sharing and comparability of data across professions and jurisdictions. Extensive attempts to develop this language and classify patient safety incidents have already been made (Davies et al., 2003; World Health Organization, 2009), albeit with limited meaningful uptake. In light of this, there is an opportunity for legislation to help strengthen communication and learning through wider use of standardised terms. In Canada, the National System for Incident Reporting may be an appropriate starting point (Canadian Institute for Health Information, 2019).

Regardless of how patient safety concerns are brought forward - whether via mandatory reporting, voluntary reporting or complaints - a learning system would allow a health system to build on what it knows and appropriately respond to a patient safety incident, share information about it and thus contribute to preventing further patient safety incidents (Baker et al., 2008; Incident Analysis Collaborating Parties, 2012). Yet, as the results of this study suggest, a robust patient safety culture, in which shame and blame are replaced with trust and proactive identification and resolution of system weaknesses, is seen as a compulsory underpinning of a learning system (Incident Analysis Collaborating Parties, 2012; Frankel et al., 2017) and overall system improvement (World Health Organization, 2009). The expert informants involved in our case study described patient safety culture as encompassing reporting, learning and sharing, consistent with a multidimensional notion of patient safety culture (Canadian Patient Safety Institute, 2016b). They also described this culture as a necessary accompaniment to mandatory reporting legislation. Their views are further supported by literature suggesting that a healthy organisational culture around patient safety incident reporting would promote the interpretation of mandatory reporting legislation and regulation in positive light and help to move patient safety culture forward as an everyday practice (Frankel et al., 2017). Legislation simply cannot create or sustain patient safety culture on its own (Small and Barach, 2002).

Although legislation cannot work alone, it can and arguably should reflect a systems governance approach. Systems governance-oriented patient safety legislation would contain provisions for investigation and analysis by experts, maintenance of a system-wide repository of data and regular review and sharing of lessons learnt (Baker et al., 2008). Just as legislation is used to create an environment where individuals feel protected to report patient safety incidents, it could also be used to extend reporting systems beyond the hospital to all health care and community care settings, or to make reporting systems accessible to a larger group of reporters than is currently the case. Governments can strengthen patient safety legislation by extending the range of its intended outcomes from merely knowing about patient safety incidents to responding and learning.

This scoping review and case study suggest that for legislation to positively impact patient safety, it must be linked with other levers throughout the system. Although our study provides a point of departure for future examinations of mandatory reporting legislation and patient safety, our findings echo work in other settings on broader issues of health care quality improvement. Leatherman and Sutherland (2007) developed a thoughtful approach to understanding the potential linkages between legislation, policy and other strategies, outlining an action framework for quality improvement. Their framework has been used to review National Health Service reforms in the UK (Leatherman and Sutherland, 2008) and recently adapted by the World Health Organization (2018) in their approach to developing national quality improvement strategies. 
The central premise of their framework is that quality improvement is complex and thus requires multi-lever, multi-tier interventions.

In the same vein, patient safety is a complex systems issue that demands responses at individual, organisational and system levels across all sectors of health care. Legislative frameworks must therefore take a systems governance perspective that supports a balance between individual accountability and the discovery of, and learning from, factors contributing to patient harm (Downie et al., 2006). Although it is a blunt instrument that cannot efficiently address micro-level concerns such as team communication or missed lab results, legislation is still useful to guide the development of organisational policy, professional regulation and other levers for patient safety, ensuring a collaborative approach to fulfilling all elements essential for reporting and learning from patient safety incidents.

\subsection{Limitations}

This study focussed on hospital-based acute care settings and on Canadian mandatory reporting laws at a single point in time; thus, we present a limited picture of the full range of laws that may impact patient safety. Our scoping review yielded only 11 empirical studies measuring the impact of legislation on patient harms, and the results presented across these studies were inconsistent. Grey literature was not explored. We are further limited by the narrowness of the definitions of patient safety and safety incidents that are adopted in Canada and internationally. The World Health Organization (2009) provides standardised sets of concepts, definitions and preferred terms for patient safety, but considers only physical health outcomes to classify harm severity. As a result, health care professionals reporting incidents may neglect psychological outcomes in their reports despite the potential for psychological trauma to greatly impact a patient's overall health and well-being. Patient safety also misses the important concept of cultural safety (Ramsden, 1990), which would be particularly relevant for Indigenous peoples and other systemically marginalised groups.

\section{Conclusions}

This study drew from academic literature and a case study of legislative frameworks in Canadian provinces and territories to better understand whether patient safety in hospital settings is improved by legislation for mandatory patient safety incident reporting. In summary, currently available evidence regarding any potential impact of mandatory reporting legislation on patient safety outcomes is limited.

Additional evidence in several areas is needed to better understand the contribution of legislation to patient safety outcomes and broader system improvement efforts in Canada and other countries. First, patient safety incident data and surveillance, including the maintenance of a patient safety incident registry, represent an underutilised opportunity to enable regulators, professionals and organisations to learn about patient safety incidents, respond to and learn from incidents, and prevent future harm. Data systems can be used to enhance transparency through system-wide and public reporting and to strengthen accountability of health care providers and system leaders. Second, concerns about the privacy of information and confidentiality must be balanced with the need to allow relevant parties to share information for the purposes of learning, responding to, and preventing patient safety incidents. Third, employees and other practitioners outside hospital-based settings, as well as patients and families, face legislative and structural barriers in proactively reporting patient safety incidents. An environment with a strong patient safety culture, wide access to reporting systems and clear channels of communication is foundational to knowing about patient safety incidents, developing appropriate responses and enacting a cycle of learning to prevent future harm.

This paper also highlights a need to explore what provisions effective patient safety incident reporting legislation contain. Our case study of patient safety legislation in Canada revealed 
that mandatory reporting legislation across the country is generally designed to gather information about, rather than respond to and prevent, patient safety incidents. Provisions for the development and use of learning systems that enable an effective response and information-sharing to prevent patient safety incidents are largely absent from current legislative frameworks. Our findings suggest that legislation can enable, but does not assure the effectiveness of, patient safety policies and initiatives at other system levels such as health care institutions. Legislation is one patient safety lever among many that exist at different levels of health care systems.

There is room to strengthen legislation by extending its focus beyond knowing about patient safety incidents to effectively responding to and preventing further incidents. Well-crafted legislation has potential to play a major role in improving patient safety by providing a framework for evidence-based interventions as well as learning and change at organisational and system levels. Where legislation has strong linkages with other levers throughout the health care system, policy can be translated into local action supported by organisational resources and capabilities. This study serves as a starting point for future research examining a wide range of perspectives, including those of governments, providers, patients and families, on the optimal use of legislation as well as its impacts on patient safety.

Supplementary material. The supplementary material for this article can be found at https://doi.org/10.1017/ S1744133121000050.

\section{References}

Arksey H and O'Malley L (2005) Scoping studies: towards a methodological framework. International Journal of Social Research Methodology 8, 19-32.

Baker GR, Norton PG, Flintoft V, Brown A, Cox J, Etchells E, Ghali WA, Hébert P, Majumdar SR, O'Beirne M, PalaciosDerflingher L, Reid RJ, Sheps S and Tamblyn R (2004) The Canadian Adverse Events Study: the incidence of adverse events among hospital patients in Canada. Canadian Medical Association Journal 170, 1678-1686.

Baker GR, Grosso F, Heinz C, Sharpe G, Beardwood J, Fabiano D, Jeffs L, McIvor P and Parsons D (2008) Review of Provincial, Territorial and Federal Legislation and Policy Related to the Reporting and Review of Adverse Events in Healthcare in Canada [November 2007]. Edmonton, Canada: Canadian Patient Safety Institute.

Berwick D (2013) A Promise to Learn, A Commitment to Act: Improving the Safety of Patients in England. London, UK: Crown Publishing.

Boucaud S and Dorschner D (2016) Patient safety incident reporting: current trends and gaps within the Canadian health system. Healthcare Quarterly 18, 66-71.

Canadian Institute for Health Information (2019) National System for Incident Reporting (NSIR). Ottawa, Canada: Canadian Institute for Health Information. Available at https://www.cihi.ca/en/national-system-for-incident-reportingnsir.

Canadian Institute for Health Information and Canadian Patient Safety Institute (2016) Measuring Patient Harm in Canadian Hospitals. Ottawa, Canada: CIHI.

Canadian Patient Safety Institute (2016a) Global Patient Safety Alerts. Edmonton, Canada: Canadian Patient Safety Institute. Available at https://www.patientsafetyinstitute.ca/en/NewsAlerts/Alerts/Pages/default.aspx.

Canadian Patient Safety Institute (2016b) Patient Safety and Incident Management Toolkit. Edmonton, Canada: Canadian Patient Safety Institute. Available at https://www.patientsafetyinstitute.ca/en/toolsResources/PatientSafetyIncidentManage mentToolkit/PatientSafetyManagement/Pages/Patient-Safety-Culture.aspx.

Canadian Patient Safety Institute (2018) Background Paper on Mandatory Reporting of Patient Safety Incidents [Draft]. Edmonton, Canada: Canadian Patient Safety Institute.

Chassin MR and Loeb JM (2013) High-reliability health care: getting there from here. The Milbank Quarterly 91, 459-490.

Daneman N, Stukel TA, Ma X, Vermeulen M and Guttmann A (2012) Reduction in Clostridium difficile infection rates after mandatory hospital public reporting: findings from a longitudinal cohort study in Canada. PLoS Medicine 9, e1001268.

Davies JM, Hébert PC and Hoffman C (2003) The Canadian Patient Safety Dictionary. Edmonton \& Ottawa, Canada: Canadian Patient Safety Institute \& Royal College of Physicians and Surgeons of Canada.

De Savigny D and Adam T (2009) Systems Thinking for Health Systems Strengthening. Geneva, Switzerland: World Health Organization.

Donaldson MS, Corrigan JM and Kohn LT (2000) To Err is Human: Building a Safer Health System. Washington, USA: National Academies Press. 
Downie J, Lahey W, Ford D, Gibson E, Thomson M, Ward T, McDonald F and Shea A (2006) Patient Safety Law: From Silos to Systems. Ottawa, Canada: Health Policy Research Program, Health Canada.

Erdmann R (2018) Learning from Others: Discovering the Key Success Factors of Patient Safety Programs Around the World [Draft]. Edmonton, Canada: Canadian Patient Safety Institute.

Flott K, Fontana G and Darzi A (2019) The Global State of Patient Safety. London, UK: Imperial College London.

Frankel A, Haraden C, Federico F and Lenoci-Edwards J (2017) A Framework for Safe, Reliable, and Effective Care, White Paper. Cambridge, USA: Institute for Healthcare Improvement and Safe \& Reliable Healthcare.

Haustein T, Gastmeier P, Holmes A, Lucet J-C, Shannon RP, Pittet D and Harbarth S (2011) Use of benchmarking and public reporting for infection control in four high-income countries. The Lancet Infectious Diseases 11, 471-481.

Health Canada (2019) Mandatory Reporting of Serious Adverse Drug Reactions and Medical Device Incidents by Hospitals: Guidance Document. Ottawa, Canada: Her Majesty the Queen in Right of Canada, as represented by the Minister of Health.

Health Quality Council of Alberta (2017) Healthcare Quality and Safety Management: A Framework for Albertans. Calgary, Canada: Health Quality.

Incident Analysis Collaborating Parties (2012) Canadian Incident Analysis Framework. Edmonton, Canada: Canadian Patient Safety Institute.

Jha AK, Larizgoitia I, Audera-Lopez C, Prasopa-Plaizier N, Waters H and Bates DW (2013) The global burden of unsafe medical care: analytic modelling of observational studies. BMJ Quality \& Safety 22, 809-815.

Leape LL and Berwick DM (2005) Five years after to err is human: what have we learned? JAMA 293, 2384-2390.

Leatherman S and Sutherland K (2007) Designing national quality reforms: a framework for action. International Journal for Quality in Health Care 19, 334-340.

Leatherman S and Sutherland K (2008) The Quest for Quality: Refining the NHS Reforms. A Policy Analysis and Chartbook. London, UK: The Nuffield Trust.

Levac D, Colquhoun H and O'Brien KK (2010) Scoping studies: advancing the methodology. Implementation Science 5, 69.

Linkin DR, Fishman NO, Shea JA, Yang W, Cary MS and Lautenbach E (2013) Public reporting of hospital-acquired infections is not associated with improved processes or outcomes. Infection Control \& Hospital Epidemiology 34, 844-846.

Lucet J-C, Parneix P, Grandbastien B and Berthelot P (2013) Should public reporting be made for performance indicators on healthcare-associated infections? Médecine et Maladies Infectieuses 43, 108-113.

Marsteller JA, Hsu Y-J and Weeks K (2014) Evaluating the impact of mandatory public reporting on participation and performance in a program to reduce central line-associated bloodstream infections: evidence from a national patient safety collaborative. American Journal of Infection Control 42, S209-S215.

Morin A and Laupacis A (2014) QCIPA Review Committee Recommendations. Toronto, Canada: QCIPA Review Committee.

National Academies of Sciences (2018) Crossing the Global Quality Chasm: Improving Health Care Worldwide. Washington, USA: National Academies Press.

OECD (2019) Health Statistics Database. Paris, France: OECD. Available at https://stats.oecd.org/index.aspx? r=513451\#.

Pakyz AL and Edmond MB (2013) Influence of state laws mandating reporting of healthcare-associated infections: the case of central line-associated bloodstream infections. Infection Control \& Hospital Epidemiology 34, 780-784.

Pellegrini C (2014) Canada Ranks low on patient safety in international comparison. Canadian Medical Association Journal 186, E12-E12.

Ramsden I (1990) Cultural safety. The New Zealand Nursing Journal 83, 18-19.

Reason J (1997) Managing the Risks of Organizational Accidents. London, UK: Routledge.

RiskAnalytica (2017) The Case for Investing in Patient Safety.

Seventy-Second World Health Assembly (2019) Patient Safety: Global Action on Patient Safety. Report by the Director-General. Geneva, Switzerland: World Health Organization.

Slawomirski L, Auraaen A and Klazinga NS (2017) The Economics of Patient Safety: Strengthening A Value-Based Approach to Reducing Patient Harm at National Level. Paris, France: Organisation for Economic Co-operation and Development (OECD).

Small SD and Barach P (2002) Patient safety and health policy: a history and review. Hematology and Oncology Clinics of North America 16, 1463-1482.

Stone PW, Horan TC, Shih H-C, Mooney-Kane C and Larson E (2007) Comparisons of health care-associated infections identification using two mechanisms for public reporting. American Journal of Infection Control 35, 145-149.

Stone PW, Pogorzelska M, Graham D, Jia H, Uchida M and Larson EL (2011) California hospitals response to state and federal policies related to health care-associated infections. Policy, Politics, \& Nursing Practice 12, 73-81.

Stone PW, Pogorzelska-Maziarz M, Reagan J, Merrill JA, Sperber B, Cairns C, et al. (2015) Impact of laws aimed at healthcare-associated infection reduction: a qualitative study. BMJ Quality \& Safety 24, 637-644.

Wachter RM (2010) Patient safety at ten: unmistakable progress, troubling gaps. Health Affairs 29, 165-173.

Waddell K, Moat KA and Lavis JN (2017) Citizen Brief: Modernizing the Oversight of the Health Workforce in Ontario.

Woodward B and Umberger R (2016) Review of best practices for CLABSI prevention and the impact of recent legislation on CLABSI reporting. Sage Open 6. doi: 2158244016677747. 
World Health Organization (2009) More than Words: Conceptual Framework for the International Classification for Patient Safety, Version 1.1, Final Technical Report. Geneva, Switzerland: World Health Organization. Available at https://www. who.int/patientsafety/taxonomy/icps_full_report.pdf.

World Health Organization (2018) Handbook for National Quality Policy and Strategy: A Practical Approach for Developing Policy and Strategy to Improve Quality of Care. Geneva, Switzerland: World Health Organization.

World Health Organization (2019) Patient Safety. Geneva, Switzerland: World Health Organization. Available at https:// www.who.int/patientsafety/en/.

Cite this article: Milligan C, Allin S, Farr M, Farmanova E, Peckham A, Byrd J, Misfeldt R, Baker GR, Marchildon GP (2021). Mandatory reporting legislation in Canada: improving systems for patient safety? Health Economics, Policy and Law 16, 355-370. https://doi.org/10.1017/S1744133121000050 